\title{
Structure and bimodules of simple Hom-alternative algebras
}

\author{
S. ATtAN \\ Département de Mathématiques, Université d'Abomey-Calavi \\ 01 BP 4521, Cotonou 01, Bénin \\ syltane2010@yahoo.fr
}

Received June 11, 2020

Accepted March 03, 2021

Presented by Rosa M. Navarro

Abstract: This paper is mainly devoted to the study of the structure of Hom-alternative algebras. Equivalent conditions for Hom-alternative algebras being solvable, simple and semi-simple are provided. Moreover, some results about Hom-alternative bimodule are found.

Key words: Bimodules, solvable, simple, Hom-alternative algebras.

MSC (2020): 13B10, 13D20, 17A30, 17D15.

\section{INTRODUCTION}

Hom-algebras are new classes of algebras which have been studied extensively in the literature during the last decade. They are algebras where the identities defining the structure are twisted by a homomorphism and began with Hom-Lie algebras [6, 9, 10, 11], motivated by quasi-deformations of Lie algebras of vector fields, in particular q-deformations of Witt and Virasoro algebras. Hom-associative algebras were introduced in [14] while Hom-alternative and Hom-Jordan algebras are introduced in [13, 20] and deformations of Homalternative and Hom-Malcev algebras are studied in [5].

Questions on the structure of simple algebras in this or that variety are one of the main questions in the theory of rings. This question, for alternative algebras, has been studied by many authors. It turns out that the only simple alternative algebras which are not associative are 8-dimensional algebras over their centers which are generalizations of the original algebra of Cayley numbers [15, 22, 23. Hence, all semisimple alternative algebras are known. Similarly, it is relevant to study simple Hom-algebras in Hom-algebras theory. In [4], the authors gave a classification theorem about multiplicative simple Hom-Lie algebras. Inspired by this study, a classification of multiplicative simple Hom-Jordan algebras is obtained in [18.

ISSN: 0213-8743 (print), 2605-5686 (online)

(C) The author(s) - Released under a Creative Commons Attribution License (CC BY-NC 3.0) 
Representations (or bimodules) and deformations are important tools in most parts of Mathematics and Physics. By means of the representation theory, we would be more aware of the corresponding algebras. The study of bimodules of Jordan algebras was initiated by N. Jacobson [7]. Subsequently the alternative case was considered by Schafer [16]. Similarly, it is very important to study representations of Hom-algebras. Fortunately, representations of Hom-Lie algebras were introduced and studied in [17], see also [1, 3]. Later the one of Hom-Jordan and Hom-alternative is presented in [2] where some useful results are obtained. Moreover representations of simple Hom-Lie algebras [4, 12] and the one of simple Hom-Jordan algebras [18] are introduced and studied in detail. In this paper, based on [18] and on [21, we will study structure and bimodules over simple Hom-alternative algebras.

The paper is organized as follows: In section two, we give the basics about Hom-alternative algebras and provide some new properties. Section three deals with the study of simple and solvable Hom-alternative algebras. Some useful results are obtained (see Lemma 3.8 and Theorem 3.9). We also mainly prove relevant theorems which are about solvability, simplicity and semi-simplicity of Hom-alternative algebras (see Theorem 3.14. Theorem 3.16 and Theorem 3.20). In section four, we prove Theorem 4.5 which is very important. It deals with the relationship between bimodules over Homalternative algebras of alternative type and the ones over their induced alternative algebras. Moreover, some relevant propositions about bimodules over Hom-alternative algebras are also displayed as applications of Theorem 4.5.

All vector spaces are assumed to be over a fixed ground field $\mathbb{K}$ of characteristic 0 .

\section{Preliminaries}

We recall some basic notions, introduced in [6, 14, 19, related to Homalgebras. For the map $\mu: A^{\otimes 2} \rightarrow A$, we will write sometimes $\mu(a \otimes b)$ as $\mu(a, b)$ or $a b$ for $a, b \in A$.

Definition 2.1. A Hom-module is a pair $\left(M, \alpha_{M}\right)$ consisting of a $\mathbb{K}$-module $M$ and a linear self-map $\alpha_{M}: M \rightarrow M$. A morphism $f:\left(M, \alpha_{M}\right) \rightarrow\left(N, \alpha_{N}\right)$ of Hom-modules is a linear map $f: M \rightarrow N$ such that $f \circ \alpha_{M}=\alpha_{N} \circ f$.

Definition 2.2. ([14, 19]) A Hom-algebra is a triple $(A, \mu, \alpha)$ in which $(A, \alpha)$ is a Hom-module, $\mu: A^{\otimes 2} \rightarrow A$ is a linear map. The Hom-algebra 
( $A, \mu, \alpha)$ is said to be multiplicative if $\alpha \circ \mu=\mu \circ \alpha^{\otimes 2}$ (multiplicativity). A morphism $f:\left(A, \mu_{A}, \alpha_{A}\right) \rightarrow\left(B, \mu_{B}, \alpha_{B}\right)$ of Hom-algebras is a morphism of the underlying Hom-modules such that $f \circ \mu_{A}=\mu_{B} \circ f^{\otimes 2}$.

In this paper, we will only consider multiplicative Hom-algebras.

Definition 2.3. Let $(A, \mu, \alpha)$ be a Hom-algebra and $\lambda \in \mathbb{K}$. Let $R$ be a linear map satisfying

$$
\mu(R(x), R(y))=R(\mu(R(x), y)+\mu(x, R(y))+\lambda \mu(x, y)), \quad \forall x, y \in A .
$$

Then, $R$ is called a Rota-Baxter operator of weight $\lambda$ and $(A, \mu, \alpha, R)$ is called a Rota-Baxter Hom-algebra of weight $\lambda$.

Definition 2.4. Let $(A, \mu, \alpha)$ be a Hom-algebra.

1. The Hom-associator of $A$ is the linear map as $A: A^{\otimes 3} \rightarrow A$ defined as $\operatorname{as}_{A}=\mu \circ(\mu \otimes \alpha-\alpha \otimes \mu)$. A multiplicative Hom-algebra $(A, \mu, \alpha)$ is said to be Hom-associative algebra if $\operatorname{as}_{A}=0$.

2. A Hom-alternative algebra [13] is a multiplicative Hom-algebra $(A, \mu, \alpha)$ that satisfies both

$$
\begin{aligned}
& \operatorname{as}_{A}(x, x, y)=0 \quad \text { (left Hom-alternativity), } \\
& \operatorname{as}_{A}(x, y, y)=0 \quad \text { (right Hom-alternativity) }
\end{aligned}
$$

for all $x, y \in A$.

3. Let $(A, \mu, \alpha)$ be a Hom-alternative algebra; a Hom-subalgebra of $(A, \mu, \alpha)$ is a linear subspace $H$ of $A$, which is closed for the multiplication $\mu$ and invariant by $\alpha$, that is, $\mu(x, y) \in H$ and $\alpha(x) \in H$ for all $x, y \in H$. If furthermore $\mu(a, b) \in H$ and $\mu(b, a) \in H$ for all $(a, b) \in A \times H$, then $H$ is called a two-sided Hom-ideal of $A$.

Example 2.5. The octonions algebra $\mathbb{O}$, also called Cayley Octaves or Cayley algebra is 8-dimensional, with a basis $\left(e_{0}, e_{1}, e_{2}, e_{3}, e_{4}, e_{5}, e_{6}, e_{7}\right)$, where $e_{0}$ is the identity for the multiplication. This algebra is twisted into the eightdimensional Hom-alternative algebra $\mathbb{O}_{\alpha}=\left(\mathbb{O}, \mu_{1}, \alpha\right)$ [20] with the same basis $\left(e_{0}, e_{1}, e_{2}, e_{3}, e_{4}, e_{5}, e_{6}, e_{7}\right)$ where

$$
\begin{array}{llll}
\alpha\left(e_{0}\right)=e_{0}, & \alpha\left(e_{1}\right)=e_{5}, & \alpha\left(e_{2}\right)=e_{6}, & \alpha\left(e_{3}\right)=e_{7}, \\
\alpha\left(e_{4}\right)=e_{1}, & \alpha\left(e_{5}\right)=e_{2}, & \alpha\left(e_{6}\right)=e_{3}, & \alpha\left(e_{7}\right)=e_{4},
\end{array}
$$


and the multiplication table is:

\begin{tabular}{|c|c|c|c|c|c|c|c|c|}
\hline$\mu_{1}$ & $e_{0}$ & $e_{1}$ & $e_{2}$ & $e_{3}$ & $e_{4}$ & $e_{5}$ & $e_{6}$ & $e_{7}$ \\
\hline$e_{0}$ & $e_{0}$ & $e_{5}$ & $e_{6}$ & $e_{7}$ & $e_{1}$ & $e_{2}$ & $e_{3}$ & $e_{4}$ \\
\hline$e_{1}$ & $e_{5}$ & $-e_{0}$ & $e_{1}$ & $e_{4}$ & $-e_{6}$ & $e_{3}$ & $-e_{2}$ & $-e_{7}$ \\
\hline$e_{2}$ & $e_{6}$ & $-e_{1}$ & $-e_{0}$ & $e_{2}$ & $e_{5}$ & $-e_{7}$ & $e_{4}$ & $-e_{3}$ \\
\hline$e_{3}$ & $e_{7}$ & $-e_{4}$ & $-e_{2}$ & $-e_{0}$ & $e_{3}$ & $e_{6}$ & $-e_{1}$ & $e_{5}$ \\
\hline$e_{4}$ & $e_{1}$ & $e_{6}$ & $-e_{5}$ & $-e_{3}$ & $-e_{0}$ & $e_{4}$ & $e_{7}$ & $-e_{2}$ \\
\hline$e_{5}$ & $e_{2}$ & $-e_{3}$ & $e_{7}$ & $-e_{6}$ & $-e_{4}$ & $-e_{0}$ & $e_{5}$ & $e_{1}$ \\
\hline$e_{6}$ & $e_{3}$ & $e_{2}$ & $-e_{4}$ & $e_{1}$ & $-e_{7}$ & $-e_{5}$ & $-e_{0}$ & $e_{6}$ \\
\hline$e_{7}$ & $e_{4}$ & $e_{7}$ & $e_{3}$ & $-e_{5}$ & $e_{2}$ & $-e_{1}$ & $-e_{6}$ & $-e_{0}$ \\
\hline
\end{tabular}

and into the eight-dimensional Hom-alternative algebra $\mathbb{O}_{\beta}=\left(\mathbb{O}, \mu_{2}, \beta\right)$ [13] with the same basis $\left(e_{0}, e_{1}, e_{2}, e_{3}, e_{4}, e_{5}, e_{6}, e_{7}\right)$ where $\beta(e i)=e i$ for all $i \in\{0,4,5,7\}$ and $\beta(e i)=-e i$ for all $i \in\{1,2,3,6\}$. The multiplication table is:

\begin{tabular}{|c|c|c|c|c|c|c|c|c|}
\hline$\mu_{2}$ & $e_{0}$ & $e_{1}$ & $e_{2}$ & $e_{3}$ & $e_{4}$ & $e_{5}$ & $e_{6}$ & $e_{7}$ \\
\hline$e_{0}$ & $e_{0}$ & $-e_{1}$ & $-e_{2}$ & $-e_{3}$ & $e_{4}$ & $e_{5}$ & $-e_{6}$ & $e_{7}$ \\
\hline$e_{1}$ & $-e_{1}$ & $-e_{0}$ & $e_{4}$ & $e_{7}$ & $e_{2}$ & $-e_{6}$ & $-e_{5}$ & $e_{3}$ \\
\hline$e_{2}$ & $-e_{2}$ & $-e_{4}$ & $-e_{0}$ & $e_{5}$ & $-e_{1}$ & $e_{3}$ & $e_{7}$ & $e_{6}$ \\
\hline$e_{3}$ & $-e_{3}$ & $-e_{7}$ & $-e_{5}$ & $-e_{0}$ & $-e_{6}$ & $-e_{2}$ & $-e_{4}$ & $-e_{1}$ \\
\hline$e_{4}$ & $e_{4}$ & $-e_{2}$ & $e_{1}$ & $e_{6}$ & $-e_{0}$ & $e_{7}$ & $-e_{3}$ & $-e_{5}$ \\
\hline$e_{5}$ & $e_{5}$ & $e_{6}$ & $-e_{3}$ & $e_{2}$ & $-e_{7}$ & $-e_{0}$ & $-e_{1}$ & $e_{4}$ \\
\hline$e_{6}$ & $-e_{6}$ & $e_{5}$ & $-e_{7}$ & $e_{4}$ & $e_{3}$ & $e_{1}$ & $-e_{0}$ & $-e_{2}$ \\
\hline$e_{7}$ & $e_{7}$ & $-e_{3}$ & $-e_{6}$ & $e_{1}$ & $e_{5}$ & $-e_{4}$ & $e_{2}$ & $-e_{0}$ \\
\hline
\end{tabular}

Nor $\mathbb{O}_{\alpha}$, neither $\mathbb{O}_{\beta}$, are alternative algebras. Moreover, both $\alpha$ and $\beta$ are automorphisms of $\mathbb{O}$.

Similarly as in [13], it easy to prove the following:

Proposition 2.6. Let $(A, \mu, \alpha)$ be a Hom-alternative algebra and $\beta$ : $A \rightarrow A$ be a morphism of $(A, \mu, \alpha)$. Then $(A, \beta \circ \mu, \beta \circ \alpha)$ is a Hom-alternative algebra. In particular, if $(A, \mu)$ is an alternative algebra and $\beta$ is a morphism of $(A, \mu)$, then $(A, \beta \circ \mu, \beta)$ is a Hom-alternative algebra [13].

Definition 2.7. Let $(A, \mu, \alpha)$ be a Hom-alternative algebra. If there is an alternative algebra $\left(A, \mu^{\prime}\right)$ such that $\mu=\alpha \circ \mu^{\prime}$, we say that $(A, \mu, \alpha)$ is alternative-type and $\left(A, \mu^{\prime}\right)$ is its compatible alternative algebra or the untwist of $(A, \mu, \alpha)$. 
It is noticed in 13 that a Hom-alternative algebra with an invertible twisting map has the compatible alternative algebra. More precisely, we get:

Corollary 2.8. ([13]) Let $(A, \mu, \alpha)$ be a Hom-alternative algebra where $\alpha$ is invertible then $\left(A, \mu^{\prime}=\alpha^{-1} \circ \mu\right)$ is an alternative algebra and $\alpha$ is an automorphism with respect to $\mu^{\prime}$. Hence $(A, \mu, \alpha)$ is alternative-type and $\left(A, \mu^{\prime}=\alpha^{-1} \circ \mu\right)$ is its compatible alternative algebra.

Proposition 2.9. Let $\left(A_{1}, \mu_{1}, \alpha_{1}\right)$ and $\left(A_{2}, \mu_{2}, \alpha_{2}\right)$ be Hom-alternative algebras and $\varphi:\left(A_{1}, \mu_{1}, \alpha_{1}\right) \rightarrow\left(A_{2}, \mu_{2}, \alpha_{2}\right)$ be an invertible morphism of Hom-algebras. If $\left(A_{1}, \mu_{1}, \alpha_{1}\right)$ is alternative-type and $\left(A_{1}, \mu_{1}^{\prime}\right)$ is its compatible alternative algebra then $\left(A_{2}, \mu_{2}, \alpha_{2}\right)$ is alternative-type with compatible alternative algebra $\left(A_{2}, \mu_{2}^{\prime}=\varphi \circ \mu_{1}^{\prime} \circ\left(\varphi^{-1} \otimes \varphi^{-1}\right)\right)$ such that $\varphi:\left(A_{1}, \mu_{1}^{\prime}\right) \rightarrow\left(A_{2}, \mu_{2}^{\prime}\right)$ is an algebra morphism.

Proof. First, let us prove that $\left(A_{2}, \mu_{2}^{\prime}\right)$ is an alternative algebra such that $\mu_{2}^{\prime}=\varphi \circ \mu_{1}^{\prime} \circ\left(\varphi^{-1} \otimes \varphi^{-1}\right)$. Denote by as ${ }_{1}$ and as 2 the associators of $\left(A_{1}, \mu_{1}\right)$ and $\left(A_{2}, \mu_{2}^{\prime}\right)$ respectively. Then

$$
\begin{aligned}
\operatorname{as}_{2}(u, u, v)= & \mu_{2}^{\prime}\left(\mu_{2}^{\prime}(u, u), v\right)-\mu_{2}^{\prime}\left(u, \mu_{2}^{\prime}(u, v)\right) \\
= & \varphi \circ \mu_{1}^{\prime}\left(\varphi^{-1} \circ \varphi \circ \mu_{1}^{\prime}\left(\varphi^{-1}(u), \varphi^{-1}(u)\right), \varphi^{-1}(v)\right) \\
& -\varphi \circ \mu_{1}^{\prime}\left(\varphi^{-1}(u), \varphi^{-1} \circ \varphi \circ \mu_{1}^{\prime}\left(\varphi^{-1}(u), \varphi^{-1}(v)\right)\right) \\
= & \varphi \circ \mu_{1}^{\prime}\left(\mu_{1}^{\prime}\left(\varphi^{-1}(u), \varphi^{-1}(u)\right), \varphi^{-1}(v)\right) \\
& -\varphi \circ \mu_{1}^{\prime}\left(\varphi^{-1}(u), \mu_{1}^{\prime}\left(\varphi^{-1}(u), \varphi^{-1}(v)\right)\right) \\
= & \varphi \circ \operatorname{as}_{1}\left(\varphi^{-1}(u), \varphi^{-1}(u), \varphi^{-1}(v)\right)=\varphi(0)=0 .
\end{aligned}
$$

Similarly, we prove that $\operatorname{as}_{2}(u, v, v)=0$. Hence $\left(A, \mu_{2}^{\prime}\right)$ is an alternative algebra.

Next, we have $\alpha_{2} \circ \varphi=\varphi \circ \alpha_{1}$ and $\varphi$ defines $\mu_{2}$ by $\mu_{2} \circ \varphi^{\otimes 2}=\varphi \circ \mu_{1}$ since $\varphi$ is a morphism from $\left(A_{1}, \mu_{1}, \alpha_{1}\right)$ to $\left(A_{2}, \mu_{2}, \alpha_{2}\right)$, i.e.,

$$
\begin{aligned}
\mu_{2} & =\varphi \circ \mu_{1} \circ\left(\varphi^{-1} \otimes \varphi^{-1}\right) \\
& =\varphi \circ \alpha_{1} \circ \mu_{1}^{\prime} \circ\left(\varphi^{-1} \otimes \varphi^{-1}\right)=\alpha_{2} \circ \varphi \circ \mu_{1}^{\prime} \circ\left(\varphi^{-1} \otimes \varphi^{-1}\right) .
\end{aligned}
$$

Hence, let take $\mu_{2}^{\prime}=\varphi \circ \mu_{1}^{\prime} \circ\left(\varphi^{-1} \otimes \varphi^{-1}\right)$.

Finally, the fact that $\varphi:\left(A_{1}, \mu_{1}^{\prime}\right) \rightarrow\left(A_{2}, \mu_{2}^{\prime}\right)$ is an algebra morphism follows from the definition of $\mu_{2}^{\prime}$. 
The following characterization was given for Hom-Lie algebras in [17] and Hom-associative algebras in [21].

Proposition 2.10. Given two Hom-alternative algebras $\left(A, \mu_{A}, \alpha\right)$ and $\left(B, \mu_{B}, \beta\right)$, there is a Hom-alternative algebra $\left(A \oplus B, \mu_{A \oplus B}, \alpha+\beta\right)$, where the bilinear map $\mu_{A \oplus B}:(A \oplus B)^{\times 2} \rightarrow(A \oplus B)$ is given by

$$
\mu_{A \oplus B}\left(a_{1}+b_{1}, a_{2}+b_{2}\right)=\mu_{A}\left(a_{1}, a_{2}\right)+\mu_{B}\left(b_{1}, b_{2}\right) \quad \forall a_{1}, a_{2} \in A, \forall b_{1}, b_{2} \in B,
$$

and the linear map $(\alpha+\beta):(A \oplus B) \rightarrow(A \oplus B)$ is given by

$$
(\alpha+\beta)(a+b)=(\alpha(a)+\beta(b)) \forall(a, b) \in A \times B .
$$

Proof. First, $(\alpha+\beta)$ is multiplicative with respect to $\mu_{A \oplus B}$. Indeed,

$$
\begin{aligned}
(\alpha+\beta) \circ( & \left.\mu_{A \oplus B}\right)\left(a_{1}+b_{1}, a_{2}+b_{2}\right) \\
& =(\alpha+\beta)\left(\mu_{A}\left(a_{1}, a_{2}\right)+\mu_{B}\left(b_{1}, b_{2}\right)\right) \\
& =\alpha \circ \mu_{A}\left(a_{1}, a_{2}\right)+\beta \circ \mu_{B}\left(b_{1}, b_{2}\right) \\
& =\mu_{A}\left(\alpha\left(a_{1}\right), \alpha\left(a_{2}\right)\right)+\mu_{B}\left(\beta\left(b_{1}\right), \beta\left(b_{2}\right)\right. \\
& =\mu_{A \oplus B}\left(\alpha\left(a_{1}\right)+\beta\left(b_{1}\right), \alpha\left(a_{2}\right)+\beta\left(b_{2}\right)\right) \\
& =\mu_{A \oplus B}\left((\alpha+\beta)\left(a_{1}+b_{1}\right),(\alpha+\beta)\left(a_{2}+b_{2}\right)\right) .
\end{aligned}
$$

Secondly we prove the left Hom-alternativity (2) for $A \oplus B$ as follows

$$
\begin{aligned}
\operatorname{as}_{A \oplus B}( & \left.a_{1}+b_{1}, a_{1}+b_{1}, a_{2}+b_{2}\right) \\
= & \mu_{A \oplus B}\left(\mu_{A \oplus B}\left(a_{1}+b_{1}, a_{1}+b_{1}\right),(\alpha+\beta)\left(a_{2}+b_{2}\right)\right) \\
& -\mu_{A \oplus B}\left((\alpha+\beta)\left(a_{1}+b_{1}\right), \mu_{A \oplus B}\left(a_{1}+b_{1}, a_{2}+b_{2}\right)\right) \\
= & \mu_{A \oplus B}\left(\mu_{A}\left(a_{1}, a_{1}\right)+\mu_{B}\left(b_{1}, b_{1}\right), \alpha\left(a_{2}\right)+\beta\left(b_{2}\right)\right) \\
& \left.-\mu_{A \oplus B}\left(\alpha\left(a_{1}\right)+\beta\left(b_{1}\right), \mu_{A}\left(a_{1}, a_{2}\right)+\mu_{(}, b_{2}\right)\right) \\
= & \mu_{A}\left(\mu_{A}\left(a_{1}, a_{1}\right), \alpha\left(a_{2}\right)\right)+\mu_{B}\left(\mu_{B}\left(b_{1}, b_{1}\right), \beta\left(b_{2}\right)\right) \\
& -\mu_{A}\left(\alpha\left(a_{1}\right), \mu_{A}\left(a_{1}, a_{2}\right)\right)-\mu_{B}\left(\beta\left(b_{1}\right), \mu_{B}\left(b_{1}, b_{2}\right)\right) \\
= & \operatorname{as}_{A}\left(a_{1}, a_{1}, a_{2}\right)+\operatorname{as}_{B}\left(b_{1}, b_{1}, b_{2}\right)=0 .
\end{aligned}
$$

Similarly, we prove the right Hom-alternativity (3) for $A \oplus B$. Hence $(A \oplus$ $\left.B, \mu_{A \oplus B}, \alpha+\beta\right)$ is a Hom-alternative algebra. 
Proposition 2.11. Let $\left(A, \mu_{A}, \alpha\right)$ and $\left(B, \mu_{B}, \beta\right)$ be two Hom-alternative algebras and $\varphi: A \rightarrow B$ be a linear map. Denote by $\Gamma_{\varphi} \subset A \oplus B$ the graph of $\varphi$. Then $\varphi$ is a morphism from the Hom-alternative algebra $\left(A, \mu_{A}, \alpha\right)$ to the Hom-alternative algebra $\left(B, \mu_{B}, \beta\right)$ if and only if its graph $\Gamma_{\varphi}$ is a Homsubalgebra of $\left(A \oplus B, \mu_{A \oplus B}, \alpha+\beta\right)$.

Proof. Let $\phi:\left(A, \mu_{A}, \alpha\right) \rightarrow\left(B, \mu_{B}, \beta\right)$ be a morphism of Hom-alternative algebras. Then we have for all $u, v \in A$,

$$
\begin{aligned}
\mu_{A \oplus B}((u, \varphi(u)),(v, \varphi(v))) & =\left(\mu_{A}(u, v), \mu_{B}(\varphi(u), \varphi(v))\right) \\
& =\left(\mu_{A}(u, v), \varphi\left(\mu_{A}(u, v)\right)\right) .
\end{aligned}
$$

Thus the graph $\Gamma_{\varphi}$ is closed under the multiplication $\mu_{A \oplus B}$. Furthermore since $\varphi \circ \alpha=\beta \circ \varphi$, we have $(\alpha \oplus \beta)(u, \varphi(u))=(\alpha(u), \beta \circ \varphi(u))=(\alpha(u), \varphi \circ \alpha(u))$, which implies that $\Gamma_{\varphi}$ is closed under $\alpha \oplus \beta$. Thus $\Gamma_{\varphi}$ is a Hom-subalgebra of $\left(A \oplus B, \mu_{A \oplus B}, \alpha \oplus \beta\right)$.

Conversely, if the graph $\Gamma_{\varphi} \subset A \oplus B$ is a Hom-subalgebra of $\left(A \oplus B, \mu_{A \oplus B}\right.$, $\alpha \oplus \beta)$, then we have

$$
\mu_{A \oplus B}((u, \varphi(u)),(v, \varphi(v)))=\left(\mu_{A}(u, v), \mu_{B}(\varphi(u), \varphi(v))\right) \in \Gamma_{\varphi},
$$

which implies that

$$
\mu_{B}(\varphi(u), \varphi(v))=\varphi\left(\mu_{A}(u, v)\right) .
$$

Furthermore, $(\alpha \oplus \beta)\left(\Gamma_{\varphi}\right) \subset \Gamma_{\varphi}$ implies

$$
(\alpha \oplus \beta)(u, \varphi(u))=(\alpha(u), \beta \circ \varphi(u)) \in \Gamma_{\varphi},
$$

which is equivalent to the condition $\beta \circ \phi(u)=\phi \circ \alpha(u)$, i.e., $\beta \circ \varphi=\varphi \circ \alpha$. Therefore, $\varphi$ is a morphism of Hom-alternative algebras.

Proposition 2.12. Let $(A, \mu, \alpha, R)$ be a Rota-Baxter Hom-alternative algebra of weight 0 such that $R$ commutes with $\alpha$. Define a new multiplication on $A$ by

$$
\mu_{R}(x, y)=\mu(R(x), y)+\mu(x, R(y)) \quad \text { for any } x, y \in A \text {. }
$$

Then $A_{R}=\left(A, \mu_{R}, \alpha\right)$ is a Hom-alternative algebra.

Proof. The multiplicativity of $\alpha$ with respect to $\mu_{R}$ follows from the one of $\alpha$ with respect to $\mu$ and the hypothesis $R \circ \alpha=\alpha \circ R$. To prove the left Hom-alternative identity, let pick $x, y \in A$. Then, 


$$
\begin{gathered}
\operatorname{as}_{R}(x, x, y)=\mu_{R}\left(\mu_{R}(x, x), \alpha(y)\right)-\mu_{R}\left(\alpha(x), \mu_{R}(x, y)\right) \\
=\mu_{R}(\mu(R(x), x)+\mu(x, R(x)), \alpha(y)) \\
\quad-\mu_{R}(\alpha(x), \mu(R(x), y)+\mu(x, R(y))) \\
=\mu(R(\mu(R(x), x)+\mu(x, R(x))), \alpha(y)) \\
\quad+\mu(\mu(R(x), x)+\mu(x, R(x)), R \circ \alpha(y)) \\
\quad-\mu(R \circ \alpha(x), \mu(R(x), y)+\mu(x, R(y))) \\
\quad-\mu(\alpha(x), R(\mu(R(x), y)+\mu(x, R(y)))) \\
=\mu(\mu(R(x), R(x)), \alpha(y))+\mu(\mu(R(x), x), \alpha \circ R(y)) \\
\quad+\mu(\mu(x, R(x)), \alpha \circ R(y))-\mu(\alpha \circ R(x), \mu(R(x), y)) \\
\quad-\mu(\alpha \circ R(x), \mu(x, R(y)))-\mu(\alpha(x), \mu(R(x), R(y))) \\
\quad(\operatorname{using}(1) \text { with } \lambda=0 \text { and } \alpha \circ R=R \circ \alpha) \\
=\operatorname{as}_{A}(R(x), R(x), y)+\operatorname{as}_{A}(R(x), x, R(y))+\operatorname{as}_{A}(x, R(x), R(y)) \\
=0+0=0 \quad(\operatorname{by}(2) \text { in } A) .
\end{gathered}
$$

Hence, we get (2) for $A_{R}$. Similarly, we prove (3) for $A_{R}$ and therefore, the conclusion follows.

\section{Structures of Hom-alternative Algebras}

In this section, we study simple and solvable Hom-alternative algebras. This study is inspired by the study given in [18] and [21]. We discuss necessary and sufficient conditions for Hom-alternative algebras to be solvable, simple and semi-simple. In the classical case, we already know that every simple alternative algebra is either an associative algebra or a Cayley-Dickson algebra over its center [15, 22]. As it turns out, there is one non-associative simple alternative algebra. Recall that the Cayley-Dickson algebras are a sequence $A_{0}, A_{1}, \ldots$ of non-associative $\mathbb{R}$-algebras with involution. The first few are familiar: $A_{0}=\mathbb{R}, A_{1}=\mathbb{C}, A_{2}=\mathbb{H}$ (the quaternions) and $A_{3}=\mathbb{O}$ (the octonions). Each algebra $A_{n}$ is constructed from the previous one $A_{n-1}$ by a doubling procedure. The first three Cayley-Dickson algebras are associative and $\mathbb{(})$ is the only one non-associative alternative Cayley-Dickson algebra. Alternativity fails in the higher Cayley-Dickson algebras. Basing on this fact, we will give in this section an example of non Hom-associative simple Homalternative algebra. 
Definition 3.1. Let $(A, \mu, \alpha)$ be a Hom-alternative algebra. Define its derived sequences as follows:

$$
\begin{aligned}
& A^{(0)}=A, \quad A^{(1)}=\mu(A, A), \quad A^{(2)}=\mu\left(A^{(1)}, A^{(1)}\right), \quad \ldots, \\
& A^{(k)}=\mu\left(A^{(k-1)}, A^{(k-1)}\right), \quad \ldots .
\end{aligned}
$$

The following elementary result will be very useful.

Lemma 3.2. Let $(A, \mu, \alpha)$ be a Hom-alternative algebra and $k \in \mathbb{N}$. Then

$$
\cdots \subseteq A^{(k+1)} \subseteq A^{(k)} \subseteq A^{(k-1)} \subseteq \cdots \subseteq A^{(2)} \subseteq A^{(1)} \subseteq A
$$

and $A^{(k)}$ is a two-sided Hom-ideal of $(A, \mu, \alpha)$.

Proof. First, it is clear that $A^{(1)} \subseteq A^{(0)}=A$. Next let $k \in \mathbb{N}$ and assume that $A^{(k)} \subseteq A^{(k-1)}$. Then, we have

$$
A^{(k+1)}=\mu\left(A^{(k)}, A^{(k)}\right) \subseteq \mu\left(A^{(k-1)}, A^{(k-1)}\right)=A^{(k)} .
$$

To prove that $A^{(k)}$ is a two-sided Hom-ideal of $(A, \mu, \alpha)$ for every $k \in \mathbb{N}$, by the inclusion condition, it suffices to prove the case $n=1$. Thanks to $A^{(1)} \subseteq A$, we have first

$$
\alpha\left(A^{(1)}\right)=\alpha(\mu(A, A))=\mu(\alpha(A), \alpha(A)) \subseteq \mu(A, A)=A^{(1)},
$$

and next

$$
\mu\left(A^{(1)}, A\right) \subseteq \mu(A, A)=A^{(1)} \quad \text { and } \quad \mu\left(A, A^{(1)}\right) \subseteq \mu(A, A)=A^{(1)} .
$$

Thus $A^{(1)}$ is a two-sided Hom-ideal of $(A, \mu, \alpha)$.

Definition 3.3. Let $(A, \mu, \alpha)$ be a Hom-alternative algebra; $(A, \mu, \alpha)$ is said to be solvable if there exists $n \in \mathbb{N}^{*}$ such that $A^{(n)}=\{0\}$.

We get the following example of solvable and non solvable Hom-alternative algebras respectively.

EXAMPLE 3.4. (a) Consider the 3-dimensional Hom-alternative algebra $(A, \mu, \alpha)$ with basis $\left(e_{1}, e_{2}, e_{3}\right)$ where $\mu\left(e_{2}, e_{2}\right)=e_{1}, \mu\left(e_{2}, e_{3}\right)=e_{1}, \mu\left(e_{3}, e_{2}\right)=$ $e_{1}, \mu\left(e_{3}, e_{3}\right)=e_{1}, \mu\left(e_{3}, e_{2}\right)=e_{1}$ and $\alpha\left(e_{1}\right)=e_{1}, \alpha\left(e_{2}\right)=e_{1}+e_{2}, \alpha\left(e_{3}\right)=$ $e_{3}$. Actually, $(A, \mu, \alpha)$ is a Hom-associative algebra (see [21], Theorem 3.6, 
Hom-algebra $\left.A_{7}^{3}\right)$. Then $A^{(1)}$ is a one-dimensional Hom-alternative algebra generated by $\left(e_{1}\right)$ defined as follows: $\mu\left(e_{1}, e_{1}\right)=0$ and $\alpha\left(e_{1}\right)=e_{1}$. It follows that $A^{(2)}=\{0\}$ and therefore $(A, \mu, \alpha)$ is solvable.

(b) Consider in the Example 2.5, the Hom-alternative algebras $\mathbb{O}_{\alpha}$ and $\mathbb{O}_{\beta}$. From their multiplication tables, we get $\left(\mathbb{O}_{\alpha}\right)^{(1)}=\mathbb{O}_{\alpha}$ and $\left(\mathbb{O}_{\beta}\right)^{(1)}=\mathbb{O}_{\beta}$. Hence for every $k \in \mathbb{N}$ we have $\left(\mathbb{O}_{\alpha}\right)^{(k)}=\mathbb{O}_{\alpha}$ and $\left(\mathbb{O}_{\beta}\right)^{(k)}=\mathbb{O}_{\beta}$. It follows that neither $\mathbb{O}_{\alpha}$ and nor $\mathbb{O}_{\beta}$ are solvable.

Definition 3.5. Let $(A, \mu, \alpha)(\alpha \neq 0)$ be a non trivial Hom-alternative algebra.

1. $(A, \mu, \alpha)$ is said to be a simple Hom-alternative algebra if $A^{(1)} \neq\{0\}$ and it has no proper two-sided Hom-ideal.

2. $(A, \mu, \alpha)$ is said to be a semi-simple Hom-alternative algebra if

$$
A=A_{1} \oplus A_{2} \oplus A_{3} \oplus \cdots \oplus A_{p}
$$

where $A_{i}(1 \leq i \leq p)$ are simple two-sided Hom-ideals of $(A, \mu, \alpha)$.

Let give the following example of non-simple Hom-alternative algebra.

EXAmple 3.6. Consider the three-dimensional Hom-alternative algebra $(A, \mu, \alpha)$ over $\mathbb{K}$ with basis $\left(e_{1}, e_{2}, e_{3}\right)$ defined by $\mu\left(e_{1}, e_{1}\right)=e_{1}, \mu\left(e_{2}, e_{2}\right)=e_{2}$, $\mu\left(e_{3}, e_{3}\right)=e_{1}, \mu\left(e_{1}, e_{3}\right)=\mu\left(e_{3}, e_{1}\right)=-e_{3}$ and $\alpha\left(e_{1}\right)=e_{1}, \alpha\left(e_{3}\right)=-e_{3}$. Actually, $(A, \mu, \alpha)$ is a Hom-associative algebra (see [21], Theorem 3.12, Homalgebra $\left.A_{3}^{\prime 3}\right)$. Consider the subspace $I=\operatorname{span}\left(e_{1}, e_{3}\right)$ of $A$. Then one can observe that $(I, \mu, \alpha)$ is a proper two-sided Hom-ideal of $(A, \mu, \alpha)$. Hence the Hom-alternative $(A, \mu, \alpha)$ is not simple.

We have the following elementary result which will be used in next sections.

Proposition 3.7. Let $(A, \mu, \alpha)$ be a simple Hom-alternative algebra. Then, for each $k \in \mathbb{N}, A^{(k)}=A$.

Proof. Thanks to the definition of $A^{(k)}$, it suffices to prove that $A^{(1)}=A$. By the simplicity of $(A, \mu, \alpha)$, we have $A^{(1)} \neq\{0\}$. Moreover by Lemma 3.2. $A^{(1)}$ is a two-sided Hom-ideal of $(A, \mu, \alpha)$ which has no proper two-sided Hom-ideal, then $A^{(1)}=A$.

The following lemma is useful for next results. 
Lemma 3.8. Let $(A, \mu, \alpha)$ be a Hom-alternative algebra. Then, $(\operatorname{Ker}(\alpha)$, $\mu, \alpha)$ is a two-sided Hom-ideal of $(A, \mu, \alpha)$.

Proof. Obvious, $\alpha(x)=0 \in \operatorname{Ker}(\alpha)$ for all $x \in \operatorname{Ker}(\alpha)$. Next, let $x, z \in A$ and $y \in \operatorname{Ker}(\alpha)$. Then $\alpha(\mu(x, y))=\mu(\alpha(x), \alpha(y))=\mu(\alpha(x), 0)=0$ and $\alpha(\mu(y, z))=\mu(\alpha(y), \alpha(z))=\mu(0, \alpha(z))=0$. Thus $\mu(x, y) \in \operatorname{Ker}(\alpha)$ and $\mu(y, z) \in \operatorname{Ker}(\alpha)$ and it follows that $(\operatorname{Ker}(\alpha), \mu, \alpha)$ is a two-sided Hom-ideal.

Proposition 3.9. Let $(A, \mu, \alpha)$ be a finite dimensional simple Homalternative algebra. Then the Hom-alternative algebra is alternative-type and $\alpha$ is an automorphism of both $(A, \mu, \alpha)$ and its induced algebra.

Proof. By Lemma 3.8, $\operatorname{Ker}(\alpha)$ is a two-sided Hom-ideal of the simple Homalternative algebra $(A, \mu, \alpha)$. Therefore $\operatorname{Ker}(\alpha)=\{0\}$ or $\operatorname{Ker}(\alpha)=A$. Since the Hom-alternative algebra is non trivial, it follows that $\operatorname{Ker}(\alpha)=\{0\}$ and $\alpha$ is an automorphism. Thus, $A$ is alternative-type (see Corollary 2.8).

Let $\left(A, \mu^{\prime}=\alpha^{-1} \circ \mu\right)$ be the induced (the compatible) alternative algebra of the simple Hom-alternative algebra $(A, \mu, \alpha)$. We have

$$
\alpha \circ \mu^{\prime}=\alpha \circ \alpha^{-1} \circ \mu=\alpha^{-1} \circ \mu \circ \alpha^{\otimes 2}=\mu^{\prime} \circ \alpha^{\otimes 2},
$$

i.e., $\alpha$ is both automorphism of $\left(A, \mu^{\prime}\right)$ and $(A, \mu, \alpha)$.

As in Hom-associative algebras case [21, by the above proposition, there exists an induced alternative algebra of any simple Hom-alternative algebra $(A, \mu, \alpha)$ and $\alpha$ is an automorphism of the induced alternative algebra. Moreover, their products are mutually determined.

Theorem 3.10. Two finite dimensional simple Hom-alternative algebras $\left(A_{1}, \mu_{1}, \alpha\right)$ and $\left(A_{2}, \mu_{2}, \beta\right)$ are isomorphic if and only if there exists an alternative algebra isomorphism $\varphi: A_{1} \rightarrow A_{2}$ (between their induced alternative algebras) which renders conjugate the two alternative algebra automorphisms $\alpha$ and $\beta$ that is $\varphi \circ \alpha=\beta \circ \varphi$.

Proof. Since $\left(A_{1}, \mu_{1}, \alpha\right)$ and $\left(A_{2}, \mu_{2}, \beta\right)$ are finite dimensional simple Homalternative algebras, they are alternative-type. Let $\left(A_{1}, \mu_{1}^{\prime}\right)$ and $\left(A_{2}, \mu_{2}^{\prime}\right)$ be their induced alternative algebras respectively. If $\varphi:\left(A_{1}, \mu_{1}, \alpha\right) \rightarrow\left(A_{2}, \mu_{2}, \beta\right)$ 
is an isomorphism of Hom-alternative algebras, then $\varphi \circ \alpha=\beta \circ \varphi$, thus $\beta^{-1} \circ \varphi=\varphi \circ \alpha^{-1}$. Moreover,

$$
\begin{aligned}
\varphi \circ \mu_{1}^{\prime} & =\varphi \circ \alpha^{-1} \circ \alpha \circ \mu_{1}^{\prime}=\varphi \circ \alpha^{-1} \circ \mu_{1} \\
& =\beta^{-1} \circ \varphi \circ \mu_{1}=\beta^{-1} \circ \mu_{2} \circ \varphi^{\otimes 2}=\mu_{2}^{\prime} \circ \varphi^{\otimes 2} .
\end{aligned}
$$

So, $\varphi$ is an isomorphism between the induced Hom-alternative algebras.

On the other hand, if there exists an isomorphism between the induced alternative algebras satisfying $\varphi \circ \alpha=\beta \circ \varphi$, then

$$
\varphi \circ \mu_{1}=\varphi \circ \alpha \circ \mu_{1}^{\prime}=\beta \circ \varphi \circ \mu_{1}^{\prime}=\beta \circ \mu_{2}^{\prime} \circ \varphi^{\otimes 2}=\mu_{2} \circ \varphi^{\otimes 2} .
$$

Let recall the following:

Proposition 3.11. ([2]) Let $(A, \mu, \alpha)$ be a Hom-alternative algebra and $I$ be a two-sided Hom-ideal of $(A, \mu, \alpha)$. Then $(A / I, \bar{\mu}, \bar{\alpha})$ is a Hom-alternative algebra where $\bar{\mu}(\bar{x}, \bar{y})=\overline{\mu(x, y)}$ and $\bar{\alpha}(\bar{x})=\alpha \overline{(x)}$ for all $\bar{x}, \bar{y} \in A / I$.

Proof. First, note that the multiplicativity of $\bar{\mu}$ with respect to $\bar{\alpha}$ follows from the one of $\mu$ with respect to $\alpha$. Next, pick $\bar{x}, \bar{y} \in A / I$. Then the left Hom-alternativity (2) in $(A / I, \bar{\mu}, \bar{\alpha})$ is proved as follows

$$
\begin{aligned}
\operatorname{as}_{A / I}(\bar{x}, \bar{x}, \bar{y}) & =\bar{u}(\bar{u}(\bar{x}, \bar{x}), \bar{\alpha}(\bar{y}))-\bar{\mu}(\bar{\alpha}(\bar{x}), \bar{\mu}(\bar{x}, \bar{y})) \\
& =\overline{\mu(\mu(x, x) \alpha(y))-\mu(\alpha(x), \mu(x, y))}=\overline{\left.\operatorname{as}_{A}(x, x, y)\right)}=\overline{0} .
\end{aligned}
$$

Hence we get (2) for $(A / I, \bar{\mu}, \bar{\alpha})$. Similarly, we get (3) and therefore $(A / I, \bar{\mu}, \bar{\alpha})$ is a Hom-alternative algebra.

Corollary 3.12. Let $(A, \mu, \alpha)$ be a finite dimensional Hom-alternative algebra such that $\alpha^{2}=\alpha$. Then, $(A / \operatorname{Ker}(\alpha), \bar{\mu}, \bar{\alpha})$ is a Hom-alternative algebra of alternative-type.

Proof. It is clear that $(A / \operatorname{Ker}(\alpha), \bar{\mu}, \bar{\alpha})$ is a Hom-alternative algebra by Proposition 3.11 since by Lemma 3.8, $\operatorname{Ker}(\alpha)$ is a two-sided Hom-ideal of $A$.

- If $\alpha$ is invertible, i.e., $\operatorname{Ker}(\alpha)=\{0\}$, then $(A / \operatorname{Ker}(\alpha), \bar{\mu}, \bar{\alpha})=(A, \mu, \alpha)$ and $(A / \operatorname{Ker}(\alpha), \bar{\mu}, \bar{\alpha})$ is a Hom-alternative algebra of alternative-type (see Corollary 2.8).

- If $\alpha$ is not invertible, then $\operatorname{Ker}(\alpha) \neq\{0\}$. Therefore we have to show that $\bar{\alpha}$ is invertible on the Hom-alternative algebra $(A / \operatorname{Ker}(\alpha), \bar{\mu}, \bar{\alpha})$. Assume 
that $\bar{x} \in \operatorname{Ker}(\bar{\alpha})$. Then $\alpha \overline{(x)}=\bar{\alpha}(\bar{x})=\overline{0}$, i.e., $\alpha(x) \in \operatorname{Ker}(\alpha)$. Since $\alpha^{2}=\alpha$, we have

$$
\alpha(x)=\alpha^{2}(x)=\alpha(\alpha(x))=0,
$$

which means that $x \in \operatorname{Ker}(\alpha)$, i.e., $\bar{x}=\overline{0}$. If follows that $\bar{\alpha}$ is invertible and thanks to the Corollary 2.8, the Hom-alternative algebra $(A / \operatorname{Ker}(\alpha), \bar{\mu}, \bar{\alpha})$ is alternative-type.

Corollary 3.13. Let $(A, \mu, \alpha)$ be a Hom-alternative algebra such that $\alpha$ is invertible. Then, $(A / \operatorname{Ker}(\alpha), \bar{\mu}, \bar{\alpha})$ is a Hom-alternative algebra of alternative-type.

TheOREm 3.14. Let $(A, \mu, \alpha)$ be a Hom-alternative algebra such that $\alpha$ is invertible. Then $(A, \mu, \alpha)$ is solvable if and only if its induced alternative algebra $\left(A, \mu^{\prime}\right)$ is solvable.

Proof. Let $(A, \mu, \alpha)$ be a Hom-alternative algebra such that $\alpha$ is invertible. Denote the derived sequences of $\left(A, \mu^{\prime}\right)$ and $(A, \mu, \alpha)$ by $A^{(i)}$ and $A_{\alpha}^{(i)}(i=$ $1,2, \cdots)$ respectively.

Suppose that $\left(A, \mu^{\prime}\right)$ is solvable. Then there exists $p \in \mathbb{N}^{*}$ such that $A^{(p)}=\{0\}$. Note that

$A_{\alpha}^{(1)}=\mu(A, A)=\alpha \circ \mu^{\prime}(A, A)=\alpha\left(A^{(1)}\right)$,

$A_{\alpha}^{(2)}=\mu\left(A_{\alpha}^{(1)}, A_{\alpha}^{(1)}\right)=\mu\left(\alpha\left(A^{(1)}\right), \alpha\left(A^{(1)}\right)\right)=\alpha^{2} \circ \mu^{\prime}\left(A^{(1)}, A^{(1)}\right)=\alpha^{2}\left(A^{(2)}\right)$,

so by induction $A_{\alpha}^{(p)}=\alpha^{p}\left(A^{(p)}\right)$. It follows that $A_{\alpha}^{(p)}=\{0\}$, which means that $(A, \mu, \alpha)$ is solvable.

On the other hand, assume that $(A, \mu, \alpha)$ is solvable. Then there exists $q \in \mathbb{N}^{*}$ such that $A_{\alpha}^{(q)}=\{0\}$. By the above proof, we get $\alpha^{q}\left(A^{(q)}\right)=$ $A_{\alpha}^{(q)}$. Note that $\alpha^{q}$ is invertible since $\alpha$ is, then $A^{(q)}=\{0\}$ that is $\left(A, \mu^{\prime}\right)$ is solvable.

Lemma 3.15. ([18]) Let $\mathcal{A}$ be an algebra over a field $\mathbb{K}$ that has the unique decomposition of direct sum of simple ideals $\mathcal{A}=\oplus_{i=1}^{s} \mathcal{A}_{i}$ where the $\mathcal{A}_{i}$ are not isomorphic to each other and $\alpha \in \operatorname{Aut}(\mathcal{A})$. Then $\alpha\left(\mathcal{A}_{i}\right)=\mathcal{A}_{i}$ $(i=1,2, \cdots, s)$. 
THEOREM 3.16. (i) Let $(A, \mu, \alpha)$ be a finite dimensional simple Homalternative algebra. Then its induced alternative algebra $\left(A, \mu^{\prime}\right)$ is semisimple. Moreover, $\left(A, \mu^{\prime}\right)$ can be decomposed into direct sum of isomorphic simple ideals. In addition, $\alpha$ acts simply transitively on simple ideals of the induced alternative algebra.

(ii) Let $\left(A, \mu^{\prime}\right)$ be a simple alternative algebra and $\alpha \in \operatorname{Aut}(A)$. Then $\left(A, \mu=\alpha \circ \mu^{\prime}, \alpha\right)$ is a simple Hom-alternative algebra.

Proof. (i) Thanks to Corollary 2.8, $\alpha$ is both automorphism with respect to $\mu^{\prime}$ and $\mu$.

Assume that $A_{1}$ is the maximal solvable two-sided ideal of $\left(A, \mu^{\prime}\right)$. Then there exists $p \in \mathbb{N}^{*}$ such that $A_{1}^{(p)}=\{0\}$. Since

$$
\begin{aligned}
\mu^{\prime}\left(A, \alpha\left(A_{1}\right)\right) & =\mu^{\prime}\left(\alpha(A), \alpha\left(A_{1}\right)\right)=\alpha\left(\mu^{\prime}\left(A, A_{1}\right)\right) \subseteq \alpha\left(A_{1}\right), \\
\mu^{\prime}\left(\alpha\left(A_{1}\right), A\right) & =\mu^{\prime}\left(\alpha\left(A_{1}\right), \alpha(A)\right)=\alpha\left(\mu^{\prime}\left(A_{1}, A\right)\right) \subseteq \alpha\left(A_{1}\right), \\
\left(\alpha\left(A_{1}\right)\right)^{(p)} & =\alpha\left(A_{1}^{(p)}\right)=\{0\},
\end{aligned}
$$

we obtain that $\alpha\left(A_{1}\right)$ is also a solvable two-sided ideal of $\left(A, \mu^{\prime}\right)$. Then $\alpha\left(A_{1}\right) \subseteq A_{1}$. Moreover

$$
\begin{aligned}
& \mu\left(A, A_{1}\right)=\alpha\left(\mu^{\prime}\left(A, A_{1}\right)\right) \subseteq \alpha\left(A_{1}\right) \subseteq A_{1}, \\
& \mu\left(A_{1}, A\right)=\alpha\left(\mu^{\prime}\left(A_{1}, A\right)\right) \subseteq \alpha\left(A_{1}\right) \subseteq A_{1} .
\end{aligned}
$$

It follows that $A_{1}$ is a two-sided Hom-ideal of $(A, \mu, \alpha)$ and we get that $A_{1}=$ $\{0\}$ or $A_{1}=A$ since $(A, \mu, \alpha)$ is simple.

If $A_{1}=A$, thanks to the proof of Theorem 3.14, we obtain

$$
A_{\alpha}^{(p)}=\alpha^{p}\left(A^{(p)}\right)=\alpha^{p}\left(A_{1}^{(p)}\right)=\{0\} .
$$

On the other hand, by the simplicity of $(A, \mu, \alpha)$, we have by Proposition 3.7 . $A_{\alpha}^{(p)}=A$, which is a contradiction. It follows that $A_{1}=\{0\}$ and thus, $\left(A, \mu^{\prime}\right)$ is semi-simple.

Now, by the semi-simplicity of $\left(A, \mu^{\prime}\right)$, we have $A=\oplus_{i=1}^{s} A_{i}$ where for all $i \in\{1, \ldots, s\}, A_{i}$ is a simple two-sided ideal of $\left(A, \mu^{\prime}\right)$. Since there may be isomorphic alternative algebras among $A_{1}, \ldots, A_{s}$, we can rewrite $A$ as follows:

$$
\begin{aligned}
A=A_{11} \oplus A_{12} \oplus \cdots \oplus A_{1 m_{1}} \oplus A_{21} \oplus A_{22} \oplus \cdots \\
\oplus A_{2 m_{2}} \oplus \cdots \oplus A_{t 1} \oplus A_{t 2} \oplus \cdots \oplus A_{t m_{t}},
\end{aligned}
$$


where $\left(A_{i j}, \mu^{\prime}\right) \cong\left(A_{i k}, \mu^{\prime}\right), 1 \leq j, k \leq m_{i}, i=1,2, \ldots, t$. Thanks to Lemma 3.15, we have

$$
\alpha\left(A_{i 1} \oplus A_{i 2} \oplus \cdots \oplus A_{i m_{i}}\right)=A_{i 1} \oplus A_{i 2} \oplus \cdots \oplus A_{i m_{i}} .
$$

Then

$$
\begin{aligned}
\mu\left(A_{i 1} \oplus A_{i 2} \oplus \cdots \oplus A_{i m_{i}}, A\right) & =\alpha\left(\mu^{\prime}\left(A_{i 1} \oplus A_{i 2} \oplus \cdots \oplus A_{i m_{i}}, A\right)\right) \\
& \subseteq \alpha\left(A_{i 1} \oplus A_{i 2} \oplus \cdots \oplus A_{i m_{i}}\right) \\
& =A_{i 1} \oplus A_{i 2} \oplus \cdots \oplus A_{i m_{i}}
\end{aligned}
$$

and

$$
\begin{aligned}
\mu\left(A, A_{i 1} \oplus A_{i 2} \oplus \cdots \oplus A_{i m_{i}}\right) & =\alpha\left(\mu^{\prime}\left(A, A_{i 1} \oplus A_{i 2} \oplus \cdots \oplus A_{i m_{i}}\right)\right) \\
& \subseteq \alpha\left(A_{i 1} \oplus A_{i 2} \oplus \cdots \oplus A_{i m_{i}}\right) \\
& =A_{i 1} \oplus A_{i 2} \oplus \cdots \oplus A_{i m_{i}}
\end{aligned}
$$

It follows that $A_{i 1} \oplus A_{i 2} \oplus \cdots \oplus A_{i m_{i}}$ are two-sided Hom-ideals of $(A, \mu, \alpha)$. Then, the simplicity of $(A, \mu, \alpha)$ implies $A_{i 1} \oplus A_{i 2} \oplus \cdots \oplus A_{i m_{i}}=\{0\}$ or $A_{i 1} \oplus A_{i 2} \oplus \cdots \oplus A_{i m_{i}}=A$. Therefore, all but one of $A_{i 1} \oplus A_{i 2} \oplus \cdots \oplus A_{i m_{i}}$ must be equal to $A$. Without lost of generality, assume that

$$
A=A_{11} \oplus A_{12} \oplus \cdots \oplus A_{1 m_{1}} .
$$

If $m_{1}=1$ then $\left(A, \mu^{\prime}\right)$ is simple. Else,

$$
\alpha\left(A_{1 p}\right)=A_{1 l} \quad\left(1 \leq l \neq p \leq m_{1}\right)
$$

since, if

$$
\alpha\left(A_{1 p}\right)=A_{1 p} \quad\left(1 \leq p \leq m_{1}\right),
$$

then, $A_{1 p}$ would be a non trivial two-sided Hom-ideal of $(A, \mu, \alpha)$ which contradicts the simplicity of $(A, \mu, \alpha)$.

In addition, it is clear that $A_{11} \oplus \alpha\left(A_{11}\right) \oplus \alpha^{2}\left(A_{11}\right) \oplus \cdots \alpha^{m_{1}-1}\left(A_{11}\right)$ is a two-sided Hom-ideal of $(A, \mu, \alpha)$. Therefore,

$$
A=A_{11} \oplus \alpha\left(A_{11}\right) \oplus \alpha^{2}\left(A_{11}\right) \oplus \cdots \oplus \alpha^{m_{1}-1}\left(A_{11}\right) .
$$

In other words, $\alpha$ acts simply transitively on simple ideals of the induced alternative algebra. 
(ii) By Proposition 2.6, it is clear that $(A, \mu, \alpha)$ is a Hom-alternative algebra.

Assume that $A_{1}$ is a non-trivial two-sided Hom-ideal of $(A, \mu, \alpha$, then we get

$$
\begin{aligned}
& \mu^{\prime}\left(A, A_{1}\right)=\alpha^{-1}\left(\mu\left(A, A_{1}\right)\right) \subseteq \alpha^{-1}\left(A_{1}\right) \subseteq A_{1}, \\
& \mu^{\prime}\left(A_{1}, A\right)=\alpha^{-1}\left(\mu\left(A_{1}, A\right)\right) \subseteq \alpha^{-1}\left(A_{1}\right) \subseteq A_{1} .
\end{aligned}
$$

It follows that $A_{1}$ is a non trivial two-sided ideal of $\left(A, \mu^{\prime}\right)$, contradiction. It follows that $(A, \mu, \alpha)$ has no trivial ideals.

If $\mu(A, A)=\{0\}$, then,

$$
\mu^{\prime}(A, A)=\alpha^{-1}(\mu(A, A))=\{0\},
$$

which is in contradicts the fact that $\left(A, \mu^{\prime}\right)$ is simple. It follows that $(A, \mu, \alpha)$ is simple.

Next, we will give an example of non Hom-associative simple Hom-alternative algebra using some results about Cayley-Dickson algebras.

Example 3.17. Consider Hom-alternative algebras $\mathbb{O}_{\alpha}=\left(\mathbb{O}, \mu_{1}, \alpha\right)$ and $\mathbb{O}_{\beta}=\left(\mathbb{O}, \mu_{2}, \beta\right)$ (see Example 2.5) obtained from the octonions algebra $\mathbb{O}$ which is an eight dimensional simple non-associative alternative algebra. Since $\alpha \in \operatorname{Aut}(\mathbb{O})$ and $\beta \in \operatorname{Aut}(\mathbb{O})$, thanks to Theorem $3.16(\mathrm{ii})$, both $\mathbb{O}_{\alpha}$ and $\mathbb{O}_{\beta}$ are eight dimensional simple Hom-alternative algebras.

Proposition 3.18. The eight dimensional simple Hom-alternative algebras $\mathbb{O}_{\alpha}$ and $\mathbb{O}_{\beta}$ are not isomorphic.

Proof. Suppose that the simple Hom-alternative algebras $\mathbb{O}_{\alpha}$ and $\mathbb{O}_{\beta}$ are isomorphic. Then by Theorem 3.10, there exists an alternative algebra isomorphism $\varphi: \mathbb{O} \rightarrow \mathbb{O}$ (between their induced alternative algebras) such that $\alpha \circ \varphi=\varphi \circ \beta$. This means by the definition of $\beta$ that for all $i \in\{1,2,3,6\}$, $\alpha\left(\varphi\left(e_{i}\right)\right)=-\varphi\left(e_{i}\right)$, i.e., $\lambda=-1$ is an eigenvalue of $\alpha$ (contradiction since the characteristic polynomial of $\alpha$ is $\left.X^{8}-X^{7}-X+1\right)$.

Remark 3.19. We know in the classical case that there exists only one example of a nonassociative simple alternative algebra that is, the CayleyDickson algebra over its center [15, 22]. In the Hom-algebra setting, Proposition 3.18, clearly proves that there is more than one simple non Homassociative Hom-alternative algebras. 
Theorem 3.20. (i) Let $(A, \mu, \alpha)$ be a finite dimensional semi-simple Hom-alternative algebra. Then $(A, \mu, \alpha)$ is alternative-type and its induced alternative algebra $\left(A, \mu^{\prime}\right)$ is also semi-simple.

(ii) Let $\left(A, \mu^{\prime}\right)$ be a semi-simple alternative algebra such that $A$ has a decomposition $A=\oplus_{i}^{s} A_{i}$ where $A_{i}(1 \leq i \leq s)$ are simple two-sided ideal of $\left(A, \mu^{\prime}\right)$. Moreover let $\alpha \in \operatorname{Aut}(A)$ satisfying $\alpha\left(A_{i}\right)=A_{i}(1 \leq i \leq s)$. Then $\left(A, \mu=\alpha \circ \mu^{\prime}, \alpha\right)$ is a semi-simple Hom-alternative algebra and has the unique decomposition.

Proof. (i) Suppose that $(A, \mu, \alpha)$ is a finite dimensional semi-simple Homalternative algebra. Then $A$ has the decomposition $A=\oplus_{i}^{s} A_{i}$ where $A_{i}(1 \leq$ $i \leq s)$ are simple two-sided Hom-ideal of $(A, \mu, \alpha)$. Then $\left(A_{i}, \mu,\left.\alpha\right|_{A_{i}}\right)(1 \leq i \leq$ $s)$ are simple finite dimensional Hom-alternative algebras. According to the proof of Proposition 3.9, $\left.\alpha\right|_{A_{i}}$ is invertible and therefore $\alpha$ is invertible. Thus thanks to Corollary 2.8, the Hom-alternative algebra $(A, \mu, \alpha)$ is alternativetype and its induced alternative algebra is $\left(A, \mu^{\prime}\right)$ with $\mu^{\prime}=\alpha^{-1} \circ \mu$.

On the other hand, by the proof of Theorem 3.16(ii), $A_{i}(1 \leq i \leq s)$ are two-sided ideal of $\left(A, \mu^{\prime}\right)$. Moreover $\left(A_{i},\left.\mu^{\prime}\right|_{A_{i}}\right)(1 \leq i \leq s)$ are induced alternative algebra of finite dimensional simple Hom-alternative algebras $\left(A_{i}, \mu,\left.\alpha\right|_{A_{i}}\right)$ $(1 \leq i \leq s)$ respectively. Thanks to Theorem 3.16 (i), $\left(A_{i}, \mu^{\prime}\right)$ are semi-simple alternative algebras and can be decomposed into direct sum of isomorphic simple two-sided ideals $A_{i}=A_{i 1} \oplus A_{i 2} \oplus \cdots \oplus A_{i m_{i}}$. It follows that $\left(A, \mu^{\prime}\right)$ is semi-simple and has the decomposition of direct sum of simple two-sided ideals

$$
\begin{aligned}
A=A_{11} \oplus A_{12} \oplus \cdots \oplus A_{1 m_{1}} \oplus A_{21} \oplus A_{22} \oplus \cdots \\
\oplus A_{2 m_{2}} \oplus \cdots \oplus A_{s 1} \oplus A_{s 2} \oplus \cdots \oplus A_{s m_{s}} .
\end{aligned}
$$

(ii) We know by Proposition 2.6 that $(A, \mu, \alpha)$ is a Hom-alternative algebra. Next, for all $1 \leq i \leq s$, the condition $\alpha\left(A_{i}\right)=A_{i}$ implies

$$
\begin{aligned}
& \mu\left(A_{i}, A\right)=\alpha\left(\mu^{\prime}\left(A_{i}, A\right)\right) \subseteq \alpha\left(A_{i}\right)=A_{i}, \\
& \mu\left(A, A_{i}\right)=\alpha\left(\mu^{\prime}\left(A, A_{i}\right)\right) \subseteq \alpha\left(A_{i}\right)=A_{i} .
\end{aligned}
$$

It follows that $A_{i}$ are two-sided Hom-ideals of $(A, \mu, \alpha)$.

If there exits non trivial two-sided Hom-ideal $A_{i_{0}}$ of $\left(A_{i}, \mu, \alpha\right)$, then we have

$$
\begin{aligned}
& \mu\left(A_{i_{0}}, A\right)=\mu\left(A_{i_{0}}, A_{1} \oplus A_{2} \oplus \cdots \oplus A_{s}\right)=\mu\left(A_{i_{0}}, A_{i}\right) \subseteq A_{i_{0}} \\
& \mu\left(A, A_{i_{0}}\right)=\mu\left(A_{1} \oplus A_{2} \oplus \cdots \oplus A_{s}, A_{i_{0}}\right)=\mu\left(A_{i_{0}}, A_{i}\right) \subseteq A_{i_{0}}
\end{aligned}
$$


It follows that $A_{i_{0}}$ is a non trivial two-sided Hom-ideal of $(A, \mu, \alpha)$. Thanks to the proof of Theorem 3.16 (ii), $A_{i_{0}}$ is also a non trivial two-sided ideal of $\left(A, \mu^{\prime}\right)$. Hence, $A_{i_{0}}$ is also a non trivial two-sided ideal of $\left(A_{i}, \mu^{\prime}\right)$ which is a contradiction. It follows that $A_{i}(i=1, \ldots, s)$ are simple two-sided Homideals of $(A, \mu, \alpha)$ and therefore $(A, \mu, \alpha)$ is semi-simple and has the unique decomposition.

Proposition 3.21. Let $(A, \mu, \alpha)$ be a Hom-alternative algebra such that $\alpha^{2}=\alpha$. Then $(A, \mu, \alpha)$ is isomorphic to the decomposition of direct sum of Hom-alternative algebras, i.e.,

$$
A \cong(A / \operatorname{Ker}(\alpha)) \oplus \operatorname{Ker}(\alpha) .
$$

Proof. It is clear that $(\operatorname{Ker}(\alpha), \mu, \alpha)$ is a Hom-alternative algebra and thanks to Proposition 3.11 since $\operatorname{Ker}(\alpha)$ is a two-sided Hom-ideal of $(A, \mu, \alpha)$, the quotient Hom-algebra $(A / \operatorname{Ker}(\alpha), \bar{\mu}, \bar{\alpha})$ is a Hom-alternative algebra. Now set $A_{1}=(A / \operatorname{Ker}(\alpha)) \oplus \operatorname{Ker}(\alpha)$ and define $\mu_{1}: A_{1}^{\times 2} \rightarrow A_{1}$ and $\alpha_{1}: A_{1} \rightarrow A_{1}$ by

$$
\mu_{1}((\bar{x}, h),(\bar{y}, k)):=(\overline{\mu(x, y)}, \mu(h, k)), \quad \alpha_{1}((\bar{x}, h)):=(\overline{\alpha(x)}, 0) .
$$

Then one can show that $\left(A_{1}, \mu_{1}, \alpha_{1}\right)$ is a Hom-alternative algebra which is a decomposition of direct sum of Hom-alternative algebras. Next, let $y \in \operatorname{Ker}(\alpha) \cap \operatorname{Im}(\alpha)$. Then, there exists $x \in A$ such that $y=\alpha(x)$. Moreover, we have

$$
0=\alpha(y)=\alpha^{2}(x)=\alpha(x)=y .
$$

It follows that $\operatorname{Ker}(\alpha) \cap \operatorname{Im}(\alpha)=\{0\}$, and then $A=\operatorname{Ker}(\alpha) \oplus \operatorname{Im}(\alpha)$ since for any $x \in A$ we have $x=(x-\alpha(x))+\alpha(x)$ with $x-\alpha(x) \in \operatorname{Ker}(\alpha)$ and $\alpha(x) \in \operatorname{Im}(\alpha)$.

Now, let show that $(\operatorname{Im}(\alpha), \mu, \alpha) \cong(V / \operatorname{Ker}(\alpha), \bar{\mu}, \bar{\alpha})$. Note that, it is clear that $(\operatorname{Im}(\alpha), \mu, \alpha)$ is a Hom-alternative algebra. Define $\varphi: V / \operatorname{Ker}(\alpha) \rightarrow$ $\operatorname{Im}(\alpha)$ by $\varphi(\bar{x})=\alpha(x)$ for all $\bar{x} \in A / \operatorname{Ker}(\alpha)$. Clearly, $\varphi$ is bijective and for all $\bar{x}, \bar{y} \in A / \operatorname{Ker}(\alpha)$, we have

$$
\begin{aligned}
\varphi(\bar{\mu}(\bar{x}, \bar{y})) & =\varphi(\overline{\mu(x, u)})=\alpha(\mu(x, y))=\mu(\alpha(x), \alpha(y))=\mu(\varphi(\bar{x}), \varphi(\bar{y})), \\
\varphi(\bar{\alpha}(\bar{x})) & =\varphi(\overline{\alpha(x)})=\alpha^{2}(x)=\alpha(\varphi(\bar{x})),
\end{aligned}
$$

i.e., $\varphi \circ \bar{\mu}=\bar{\mu} \circ \varphi^{\otimes 2}$ and $\varphi \circ \bar{\alpha}=\alpha \circ \varphi$. It follows that $(\operatorname{Im}(\alpha), \mu, \alpha) \cong$ $(V / \operatorname{Ker}(\alpha), \bar{\mu}, \bar{\alpha})$ and therefore

$$
V=\operatorname{Ker}(\alpha) \oplus \operatorname{Im}(\alpha) \cong(A / \operatorname{Ker}(\alpha)) \oplus \operatorname{Ker}(\alpha) .
$$




\section{Bimodules over simple Hom-Alternative Algebras}

In this section, we mainly study bimodules over Hom-alternative algebras of alternative-type. We give a theorem about the relationship between bimodules over Hom-alternative algebras of alternative-type and the ones over their induced alternative algebras. Moreover, some relevant propositions about bimodules over Hom-alternative algebras are also displayed. As a consequence, interesting results about bimodules over finite dimensional simple Hom-alternative algebras are given.

Definition 4.1. Let $\mathcal{A}^{\prime}=(A, \mu)$ be any algebra and $V$ be a $\mathbb{K}$-module.

1. A left (resp. right) structure map on $V$ is a morphism $\delta_{l}: A \otimes V \rightarrow V$, $a \otimes v \longmapsto a \cdot v$ (resp. $\delta_{r}: V \otimes A \rightarrow V, v \otimes a \longmapsto v \cdot a$ ) of Hom-modules.

2. Let $\delta_{l}$ and $\delta_{r}$ be structure maps on $V$. Then the module associator of $V$ is a trilinear map $(,,)_{A, V}$ defined as:

$$
\begin{aligned}
& (,,)_{A, V} \circ I d_{V \otimes A \otimes A}=\delta_{r} \circ\left(\delta_{r} \otimes I d_{A}\right)-\delta_{l} \circ\left(I d_{V} \otimes \mu\right), \\
& (,,)_{A, V} \circ I d_{A \otimes V \otimes A}=\delta_{r} \circ\left(\delta_{l} \otimes I d_{A}\right)-\delta_{l} \circ\left(I d_{A} \otimes \delta_{r}\right), \\
& (,,)_{A, V} \circ I d_{A \otimes A \otimes V}=\delta_{l} \circ\left(\mu \otimes I d_{V}\right)-\delta_{l} \circ\left(I d_{A} \otimes \delta_{l}\right) .
\end{aligned}
$$

A bimodule over alternative algebras is given in [8, 16].

Definition 4.2. ([8, 16]) Let $\mathcal{A}^{\prime}=(A, \mu)$ be an alternative algebra. An alternative $\mathcal{A}^{\prime}$-bimodule is a $\mathbb{K}$-module $V$ that comes equipped with a (left) structure map $\delta_{l}: A \otimes V \rightarrow V\left(\delta_{l}(a \otimes v)=a \cdot v\right)$ and a (right) structure map $\delta_{r}: V \otimes A \rightarrow V\left(\delta_{r}(v \otimes a)=v \cdot a\right)$ such that the following equalities holds:

$$
(a, v, b)_{A, V}=-(v, a, b)_{A, V}=(b, a, v)_{A, V}=-(a, b, v)_{A, V} .
$$

The notion of alternative bimodules has been extended to the Homalternative bimodules. More precisely, we get

Definition 4.3. ([2]) Let $\mathcal{A}=\left(A, \mu, \alpha_{A}\right)$ be a Hom-alternative algebra. A Hom-alternative $\mathcal{A}$-bimodule is a Hom-module $\left(V, \alpha_{V}\right)$ that comes equipped with a (left) structure map $\rho_{l}: A \otimes V \rightarrow V\left(\rho_{l}(a \otimes v)=a \cdot v\right)$ and a (right) structure map $\rho_{r}: V \otimes A \rightarrow V\left(\rho_{r}(v \otimes a)=v \cdot a\right)$ such that the following equalities

$$
\operatorname{as}_{A, V}(a, v, b)=-\operatorname{as}_{A, V}(v, a, b)=\operatorname{as}_{A, V}(b, a, v)=-\operatorname{as}_{A, V}(a, b, v)
$$


hold for all $a, b, c \in A$ and $v \in V$, where as $A, V$ is the module Hom-associator of the Hom-module $\left(V, \alpha_{V}\right)$ defined by

$$
\begin{aligned}
& \operatorname{as}_{A, V} \circ I d_{V \otimes A \otimes A}=\rho_{r} \circ\left(\rho_{r} \otimes \alpha_{A}\right)-\rho_{l} \circ\left(\alpha_{V} \otimes \mu\right), \\
& \operatorname{as}_{A, V} \circ I d_{A \otimes V \otimes A}=\rho_{r} \circ\left(\rho_{l} \otimes \alpha_{A}\right)-\rho_{l} \circ\left(\alpha_{A} \otimes \rho_{r}\right), \\
& \operatorname{as}_{A, V} \circ I d_{A \otimes A \otimes V}=\rho_{l} \circ\left(\mu \otimes \alpha_{V}\right)-\rho_{l} \circ\left(\alpha_{A} \otimes \rho_{l}\right) .
\end{aligned}
$$

Remark 4.4. If $\alpha_{A}=I d_{A}$ and $\alpha_{V}=I d_{V}$, the notion of Hom-alternative bimodule is reduced to the one of alternative bimodule.

THEOREM 4.5. (i) Let $\mathcal{A}=\left(A, \mu, \alpha_{A}\right)$ be a Hom-alternative algebra of alternative-type with $\mathcal{A}^{\prime}=\left(A, \mu^{\prime}\right)$ its induced alternative algebra and $\left(V, \alpha_{V}\right)$ be a Hom-alternative $\mathcal{A}$-bimodule with the structure maps $\rho_{l}$ and $\rho_{r}$ such that $\alpha_{V}$ is invertible. Then $V$ is an alternative $\mathcal{A}^{\prime}$-bimodule with the structures maps $\delta_{l}=\alpha_{V}^{-1} \circ \rho_{l}$ and $\delta_{r}=\alpha_{V}^{-1} \circ \rho_{r}$.

(ii) Let $\mathcal{A}^{\prime}=\left(A, \mu^{\prime}\right)$ be an alternative algebra and $\mathcal{A}=\left(A, \mu=\alpha_{A} \circ \mu^{\prime}, \alpha_{A}\right)$ a corresponding Hom-alternative algebra. Let $V$ be an alternative $\mathcal{A}^{\prime}$ bimodule with the structure maps $\delta_{l}$ and $\delta_{r}$ and $\alpha_{V} \in \operatorname{End}(V)$ such that $\alpha_{V} \circ \delta_{l}=\delta_{l} \circ\left(\alpha_{A} \otimes \alpha_{V}\right)$ and $\alpha_{V} \circ \delta_{r}=\delta_{r} \circ\left(\alpha_{V} \otimes \alpha_{A}\right)$. Then $\left(V, \alpha_{V}\right)$ is a Hom-alternative $\mathcal{A}$-bimodule with the structures maps $\rho_{l}=\alpha_{V} \circ \delta_{l}$ and $\rho_{r}=\alpha_{V} \circ \delta_{r}$ where $\alpha_{A}$ is a morphism of $\left(A, \mu^{\prime}\right)$.

Proof. (i) Let $\left(V, \alpha_{V}\right)$ be an alternative $\mathcal{A}$-bimodule with the structure maps $\rho_{l}$ and $\rho_{r}$ such that $\alpha_{V}$ is invertible. Note that the structure maps $\delta_{l}=\alpha_{V}^{-1} \circ \rho_{l}$ and $\delta_{r}=\alpha_{V}^{-1} \circ \rho_{r}$ satisfy conditions

$$
\alpha_{V} \circ \delta_{l}=\delta_{l} \circ\left(\alpha_{A} \otimes \alpha_{V}\right) \quad \text { and } \quad \alpha_{V} \circ \delta_{r}=\delta_{r} \circ\left(\alpha_{V} \otimes \alpha_{A}\right) .
$$

Therefore,

$$
\begin{aligned}
\operatorname{as}_{A, V} \circ I d_{A \otimes V \otimes A} & =\rho_{r} \circ\left(\rho_{l} \otimes \alpha_{A}\right)-\rho_{l} \circ\left(\alpha_{A} \otimes \rho_{r}\right) \\
& =\alpha_{V} \circ \delta_{r} \circ\left(\alpha_{V} \circ \delta_{l} \otimes \alpha_{A}\right)-\alpha_{V} \circ \delta_{l} \circ\left(\alpha_{A} \otimes \alpha_{V} \circ \delta_{r}\right) \\
& =\alpha_{V}^{2} \circ \delta_{r} \circ\left(\delta_{l} \otimes I d_{A}\right)-\alpha_{V}^{2} \circ \delta_{l} \circ\left(I d_{A} \otimes \delta_{r}\right) \quad(\text { by (7) }) \\
& =\alpha_{V}^{2} \circ(,,)_{A, V} \circ I d_{A \otimes V \otimes A} .
\end{aligned}
$$

Thus, $(,,)_{A, V} \circ I d_{A \otimes V \otimes A}=\left(\alpha_{V}^{2}\right)^{-1} \circ \operatorname{as}_{A, V} \circ I d_{A \otimes V \otimes A}$. Similarly, we get

$$
\begin{aligned}
& (,,)_{A, V} \circ I d_{A \otimes A \otimes V}=\left(\alpha_{V}^{2}\right)^{-1} \circ \operatorname{as}_{A, V} \circ I d_{A \otimes A \otimes V}, \\
& (,,)_{A, V} \circ I d_{V \otimes A \otimes A}=\left(\alpha_{V}^{2}\right)^{-1} \circ \operatorname{as}_{A, V} \circ I d_{V \otimes A \otimes A} .
\end{aligned}
$$


Finally, Condition (5) follows from Condition (6).

(ii) Similar to (i).

Corollary 4.6. Let $\mathcal{A}=\left(A, \mu, \alpha_{A}\right)$ be a finite dimensional simple Homalternative algebra and $\left(V, \alpha_{V}\right)$ be a Hom-alternative $\mathcal{A}$-bimodule with the structure maps $\rho_{l}$ and $\rho_{r}$ such that $\alpha_{V}$ is invertible. Then $V$ is an alternative $\mathcal{A}^{\prime}$-bimodule with the structures maps $\delta_{l}=\alpha_{V}^{-1} \circ \rho_{l}$ and $\delta_{r}=\alpha_{V}^{-1} \circ \rho_{r}$ where $\mathcal{A}^{\prime}=\left(A, \mu^{\prime}\right)$ is the induced alternative algebra.

Definition 4.7. Let $\mathcal{A}=\left(A, \mu, \alpha_{A}\right)$ be a Hom-alternative algebra and $\left(V, \alpha_{V}\right)$ be a Hom-alternative $\mathcal{A}$-bimodule with the structure $\rho_{l}$ and $\rho_{r}$.

1. A subspace $V_{0}$ of $V$ is called an $\mathcal{A}$-subbimodule of $\left(V, \alpha_{V}\right)$ if $\alpha_{V}\left(V_{0}\right) \subseteq V_{0}$, $\rho_{l}\left(A \otimes V_{0}\right) \subseteq V_{0}$ and $\rho_{r}\left(V_{0} \otimes A\right) \subseteq V_{0}$.

2. The Hom-alternative $\mathcal{A}$-module $\left(V, \alpha_{V}\right)$ is said to be irreducible if it has no non trivial $\mathcal{A}$-subbimodules and completely reducible if $V=$ $V_{1} \oplus V_{2} \oplus \cdots \oplus V_{s}$ where $V_{i}(1 \leq i \leq s)$ are irreducible $\mathcal{A}$-subbimodules of $\left(V, \alpha_{V}\right)$.

Proposition 4.8. Let $\mathcal{A}=\left(A, \mu, \alpha_{A}\right)$ be a Hom-alternative algebra and $\left(V, \alpha_{V}\right)$ a Hom-alternative $\mathcal{A}$-bimodule with the structure maps $\rho_{l}$ and $\rho_{r}$. Then $\operatorname{Ker}\left(\alpha_{V}\right)$ is an $\mathcal{A}$-subbimodule of $\left(V, \alpha_{V}\right)$. Moreover if $\alpha_{A}$ is surjective then $\operatorname{Im}\left(\alpha_{V}\right)$ is an $\mathcal{A}$-subbimodule of $\left(V, \alpha_{V}\right)$ and we have the isomorphism of $\mathcal{A}$-bimodules $\overline{\alpha_{V}}: V / \operatorname{Ker}\left(\alpha_{V}\right) \rightarrow \operatorname{Im}\left(\alpha_{V}\right)$.

Proof. Obvious, we have $\alpha_{V}\left(\operatorname{Ker}\left(\alpha_{V}\right)\right) \subseteq \operatorname{Ker}\left(\alpha_{V}\right)$.

Next, let $(v, a) \in \operatorname{Ker}\left(\alpha_{V}\right) \times A$. Then we get

$$
\begin{gathered}
\alpha_{V}\left(\rho_{l}(a \otimes v)\right)=\rho_{l}\left(\alpha_{A}(a) \otimes \alpha_{V}(v)\right)=0, \\
\alpha_{V}\left(\rho_{r}(v \otimes a)\right)=\rho_{r}\left(\alpha_{V}(v) \otimes \alpha_{A}(a)\right)=0
\end{gathered}
$$

since $\alpha_{V}(v)=0$. Therefore $\operatorname{Ker}\left(\alpha_{V}\right)$ is an $\mathcal{A}$-subbimodule of $\left(V, \alpha_{V}\right)$.

Similarly, it is obvious that $\alpha_{V}\left(\operatorname{Im}\left(\alpha_{V}\right)\right) \subseteq \operatorname{Im}\left(\alpha_{V}\right)$. Let $(v, a) \in \operatorname{Im}\left(\alpha_{V}\right) \times A$. Then there exits $v^{\prime} \in V$ and if $\alpha_{A}$ is surjective $a^{\prime} \in A$ such that $v=\alpha_{V}\left(v^{\prime}\right)$ and $a=\alpha_{A}\left(a^{\prime}\right)$. Therefore

$$
\begin{aligned}
& \rho_{l}(a \otimes v)=\rho_{l}\left(\alpha_{A}\left(a^{\prime}\right) \otimes \alpha_{V}\left(v^{\prime}\right)\right)=\alpha_{V}\left(\rho_{l}\left(a^{\prime} \otimes v^{\prime}\right)\right) \in \operatorname{Im}\left(\alpha_{V}\right), \\
& \rho_{r}(v \otimes a)=\rho_{r}\left(\alpha_{V}\left(v^{\prime}\right) \otimes \alpha_{A}\left(a^{\prime}\right)\right)=\alpha_{V}\left(\rho_{r}\left(v^{\prime} \otimes a^{\prime}\right)\right) \in \operatorname{Im}\left(\alpha_{V}\right) .
\end{aligned}
$$

Thus $\operatorname{Im}\left(\alpha_{V}\right)$ is an $\mathcal{A}$-subbimodule of $\left(V, \alpha_{V}\right)$. 
Finally, if define the map $\overline{\alpha_{V}}: V / \operatorname{Ker}\left(\alpha_{V}\right) \rightarrow \operatorname{Im}\left(\alpha_{V}\right)$ by $\overline{\alpha_{V}}(\bar{v})=\alpha_{V}(v)$, then it is easy to prove that $\overline{\alpha_{V}}$ is an isomorphism.

Corollary 4.9. Let $\mathcal{A}=\left(A, \mu, \alpha_{A}\right)$ be a Hom-alternative algebra and $\left(V, \alpha_{V}\right)$ be a finite dimensional irreducible Hom-alternative $\mathcal{A}$-bimodule. Then $\alpha_{V}$ is invertible.

Proposition 4.10. Let $\mathcal{A}=\left(A, \mu, \alpha_{A}\right)$ be a Hom-alternative algebra of alternative-type and $\left(V, \alpha_{V}\right)$ a Hom-alternative $\mathcal{A}$-bimodule with the structure maps $\rho_{l}$ and $\rho_{r}$ such that $\alpha_{V}$ is invertible. If the alternative $\mathcal{A}^{\prime}$-bimodule $V$ over the induced alternative algebra $\mathcal{A}^{\prime}=\left(A, \mu^{\prime}\right)$ with the structures maps $\delta_{l}=\alpha_{V}^{-1} \circ \rho_{l}$ and $\delta_{r}=\alpha_{V}^{-1} \circ \rho_{r}$ is irreducible, then the Hom-alternative $\mathcal{A}$-bimodule $\left(V, \alpha_{V}\right)$ is also irreducible.

Proof. Assume that the Hom-alternative $\mathcal{A}$-bimodule $\left(V, \alpha_{V}\right)$ is reducible. Then there exists a non trivial subspace $V_{0}$ such that $\left(V_{0},\left.\alpha_{V}\right|_{V_{0}}\right)$ is an $\mathcal{A}$-subbimodule of $\left(V, \alpha_{V}\right)$. Therefore $\left.\alpha_{V}\left(V_{0}\right) \subseteq V_{0}, \rho_{l}(a \otimes v)\right) \in V_{0}$ and $\rho_{r}(v \otimes a) \in V_{0}$ for all $(a, v) \in A \times V_{0}$. Hence $\left.\delta_{l}(a \otimes v)\right) \in \alpha_{V}^{-1}\left(V_{0}\right)=V_{0}$ and $\delta_{r}(v \otimes a) \in \alpha_{V}^{-1}\left(V_{0}\right)=V_{0}$. Thus $V_{0}$ is a non trivial $\mathcal{A}^{\prime}$-subbimodule of $V$, contradiction. It follows that $\left(V, \alpha_{V}\right)$ is an irreducible Hom-alternative $\mathcal{A}$-bimodule.

Corollary 4.11. Let $\mathcal{A}=\left(A, \mu, \alpha_{A}\right)$ be a finite dimensional simple Homalternative algebra and $\left(V, \alpha_{V}\right)$ a Hom-alternative $\mathcal{A}$-bimodule with the structure maps $\rho_{l}$ and $\rho_{r}$ such that $\alpha_{V}$ is invertible. If the alternative $\mathcal{A}^{\prime}$-bimodule $V$ over the induced alternative algebra $\mathcal{A}^{\prime}=\left(A, \mu^{\prime}\right)$ with the structures maps $\delta_{l}=\alpha_{V}^{-1} \circ \rho_{l}$ and $\delta_{r}=\alpha_{V}^{-1} \circ \rho_{r}$ is irreducible, then the Hom-alternative $\mathcal{A}$-bimodule $\left(V, \alpha_{V}\right)$ is also irreducible.

Let recall the following result from [16] which is very useful for the next result.

THEOREM 4.12. ([16]) Let $\mathcal{A}^{\prime}=\left(A, \mu^{\prime}\right)$ be a semi-simple alternative algebra. Then any representation $(S, T)$ of $\mathcal{A}^{\prime}$ is completely reducible.

Since the notion of completely reducible representation of $\mathcal{A}^{\prime}$, is equivalent to the notion of a completely reducible alternative $\mathcal{A}^{\prime}$-bimodule, thanks to Theorem 3.20 (i) and Theorem 4.5(i), we get the following important result.

Corollary 4.13. Let $\mathcal{A}=(A, \mu, \alpha)$ be a finite dimensional semi-simple Hom-alternative algebra. Then any Hom-alternative $\mathcal{A}$-bimodule $\left(V, \alpha_{V}\right)$ such that $\alpha_{V}$ is invertible, is completely reducible. 
Example 4.14. Consider the following Hom-algebra $\mathcal{A}=(A, \mu, \alpha)$ where the non-zero products are given by $\mu\left(e_{1}, e_{1}\right)=e_{1}, \mu\left(e_{2}, e_{2}\right)=e_{2}, \mu\left(e_{3}, e_{3}\right)=$ $e_{1}, \mu\left(e_{1}, e_{3}\right)=\mu\left(e_{3}, e_{1}\right)=-e_{3}$ and $\alpha\left(e_{1}\right)=e_{1}, \alpha\left(e_{3}\right)=-e_{3}$. Then $\mathcal{A}$ is a semi-simple Hom-alternative algebra with the decomposition $A_{1} \oplus A_{2}$ where $A_{1}$ and $A_{2}$ are simple two sided-Hom-ideals generated by $\left(e_{2}\right)$ and $\left(e_{1}, e_{3}\right)$ respectively. Therefore, any Hom-alternative $\mathcal{A}$-bimodule $\left(V, \alpha_{V}\right)$ such that $\alpha_{V}$ is invertible, is completely reducible.

\section{ACKNOWLEDGEMENTS}

The author would like to thank the anonymous reviewers for their valuable comments which improved the paper.

\section{REFERENCES}

[1] B. Agrebaoui, K. Benali, A. Makhlouf, Representations of simple Hom-Lie algebras, J. Lie Theory 29 (4) (2019), 1119-1135.

[2] S. Attan, H. Hounnon, B. Kpamegan, Hom-Jordan and Homalternative bimodules, Extracta Math. 35 (1) (2020), 69-97.

[3] S. Benayadi, A. Makhlouf, Hom-Lie algebras with symmetric invariant nondegenerate bilinear forms, J. Geom. Phys. 76 (2014) 38-60.

[4] X. Chen, W. HAn, Classification of multiplicative simple Hom-Lie algebras, J. Lie Theory 26 (3) (2016) 767-775.

[5] M. Elhamdadi, A. Makhlouf, Deformations of Hom-alternative and Hom-Malcev algebras, Algebras Groups Geom. 28 (2) (2011), 117-145.

[6] J.T. Hartwig, D. Larsson, S.D. Silvestrov, Deformations of Lie algebras using $\sigma$-derivations, J. Algebra 295 (2) (2006), 314-361.

[7] N. JACOBSON, General representation theory of Jordan algebras, Trans. Amer. Math. Soc. 70 (1951), 509-530.

[8] N. Jacobson, Structure of alternative and Jordan bimodules, Osaka Math. J. 6 (1954), 1-71.

[9] D. Larsson, S.D. Silvestrov, Quasi-Hom-Lie algebras, Central Extensions and 2-cocycle-like identities, J. Algebra 288 (2) (2005), 321-344.

[10] D. Larsson, S.D. Silvestrov, Quasi-Lie algebras, in "Noncommutative geometry and representation theory in mathematical physics", Contemp. Math. 391, Amer. Math. Soc., Providence, RI, 2005, 241-248.

[11] D. Larsson, S.D. Silvestrov, Quasi-deformations of $\mathfrak{s l}_{2}(\mathbb{F})$ using twisted derivations, Comm. Algebra 35 (12) (2007), 4303-4318.

[12] X.X. LI, Structures of multiplicative Hom-Lie algebras, Adv. Math. (China) $43(6)$ (2014), 817-823.

[13] A. Makhlouf, Hom-alternative algebras and Hom-Jordan algebras, Int. Electron. J. Algebra 8 (2010), 177-190. 
[14] A. Makhlouf, S.D. Silvestrov, Hom-algebra structures, J. Gen. Lie Theory Appl. 2 (2) (2008), 51-64.

[15] R.D. Schafer, Alternative algebras over an arbitrary field, Bull. Amer. Math. Soc. 49 (1943), 549-555.

[16] R.D. Schafer, Representations of alternative algebras, Trans. Amer. Math. Soc. 72 (1952), $1-17$.

[17] Y. Sheng, Representation of hom-Lie algebras, Algebr. Represent. Theory 15 (6) (2012), 1081-1098.

[18] C. Yao, Y. Ma, L. Chen, Structures, classifications and bimodules of multiplicative simple Hom-Jordan algebras, arxiv:1906.04561v1.

[19] D. YAU, Hom-algebras as deformations and homology, arxiv:0712.3515v1.

[20] D. YAU, Hom-Maltsev, Hom-alternative and Hom-Jordan algebras, Int. Electon. J. Algebra 11 (2012), $177-217$.

[21] A. Zahari, A. Makhlouf Structure and classification of Hom-associative algebras, Acta Comment. Univ. Tartu. Math. 24 (1) (2020), 79-102.

[22] M. Zorn, Theorie der alternativen Ringe, Abh. Math. Sem. Univ. Hamburg 8 (1) (1931), 123-147.

[23] M. Zorn, Alternativkörper und quadratische Systeme, Abh. Math. Sem. Univ. Hamburg 9 (1) (1933), 395-402. 